\title{
Role of Trace Elements for Oxidative Status and Quality of Human Sperm
}

\author{
Galina Nenkova1, Lubomir Petrov², Albena Alexandrova1,2 \\ ${ }^{1}$ Laboratory of Free Radical Processes, Institute of Neurobiology, Bulgarian Academy of Science, Sofia, Bulgaria \\ ${ }^{2}$ Department of Physiology and Biochemistry, National Sports Academy, Sofia, Bulgaria
}

Background: Oxidative stress affects sperm quality negatively. To maintain the pro/antioxidant balance, some metal ions (e.g. copper, zink, iron, selenium), which are co-factors of the antioxidant enzymes, are essential. However, iron and copper could act as prooxidants inducing oxidative damage of spermatozoa.

Aims: To reveal a possible correlation between the concentrations of some metal ions (iron, copper, zinc, and selenium) in human seminal plasma, oxidative stress, assessed by malondialdehyde and total glutathione levels, and semen quality, assessed by the parameters count, motility, and morphology.

Study Design: Descriptive study.

Methods: The semen analysis for volume, count, and motility was performed according to World Health Organization (2010) guidelines, using computer-assisted semen analysis. For the determination of spermatozoa morphology, a SpermBlue staining method was applied. Depending on their parameters, the sperm samples were categorized into normozoospermic, teratozoospermic, asthenoteratozoospermic, and oligoteratozoospermic. The seminal plasma content of iron, copper, zinc, and selenium was estimated by atomic absorption spectroscopy. The malondialdehyde and total glutathione levels were quantified spectrophotometrically.

Results: In the groups with poor sperm quality, the levels of $\mathrm{Fe}$ were higher, whereas those of $\mathrm{Zn}$ and $\mathrm{Se}$ were significantly lower than in the normozoospermic group. In all groups with poor sperm quality, increased levels of malondialdehyde and decreased glutathione levels were detected as evidence of oxidative stress occurrence. All these differences are most pronounced in the asthenoteratozoospermic group where values differ nearly twice as much compared to the normozoospermic group. The Fe concentration correlated positively with the malondialdehyde $(\mathrm{r}=0.666, \mathrm{p}=0.018)$, whereas it showed a negative correlation with the level of total glutathione $(r=-0.689, p=0.013)$. The total glutathione level correlated positively with the sperm motility $(\mathrm{r}=0.589, \mathrm{p}=0.044)$.

Conclusion: The elevated levels of Fe and the reduced Se levels are associated with sperm damage. The changes in the concentrations of the trace elements in human seminal plasma may be related to sperm quality since they are involved in the maintenance of the pro-/ antioxidative balance in ejaculate.

Keywords: Metal ions, oxidative status, sperm

The preliminary data of this study has been presented as oral presentation in The Eight Workshop on Biological Activity of Metals, Synthetic Compounds and Natural Products, November 27-29, 2013, Sofia, Bulgaria.

Address for Correspondence: Dr. Albena Alexandrova, Laboratory of Free Radical Processes, Institute of Neurobiology, Bulgarian Academy of Science, Sofia, Bulgaria Phone: +35929792104 e-mail: a_alexandrova_bas@yahoo.com

Received: 24 June $2016 \quad$ Accepted: 20 February 2017•DOI: 10.4274/balkanmedj.2016.0147

Available at www.balkanmedicaljournal.org

Cite this article as:

Nenkova G, Petrov L, Alexandrova A. Role of Trace Elements for Oxidative Status and Quality of Human Sperm. Balkan Med J 2017;34:343-8

${ }^{\circ}$ Copyright 2017 by Trakya University Faculty of Medicine / The Balkan Medical Journal published by Galenos Publishing House. 
Currently, infertility is becoming an increasingly widespread problem globally. In developed countries, infertility affects $3.5 \%$ to $16.7 \%$ of couples, and in less developed ones, $6.9 \%$ to $9.3 \%$ (1). Male infertility is as common as female infertility. About $30 \%$ of the issues involved with infertility are due to the man and another $30 \%$ due to the woman, while 30\% result from complications with both partners, and in a further $10 \%$ of cases, the cause is unknown. Male factor infertility is a complex disorder. Many studies indicate that oxidative stress is one of the factors that influence negatively the fertility potential of spermatozoa. Oxidative stress is defined as a disruption in the balance between reactive oxygen species (ROS) production and the ROS scavenger capacity of the antioxidant system. ROS are a product of oxidative metabolism and at physiological levels are required for normal sperm functions such as hyperactivation, capacitation, and acrosome reaction (2). However, excessive ROS can be toxic, because in exceeding the available antioxidants, these highly reactive species can react with nearby molecules and can cause DNA, protein, and lipid molecules peroxidative damage within the cell (2). In turn, oxidative alterations provoke sperm dysfunction such as a loss of motility and viability, and impairment of sperm-oocyte fusion (3). Therefore, it is essential that the antioxidant system effective in order to overcome these conditions. Seminal plasma is rich in antioxidants (4) such as vitamins C (5) and E (6), glutathione (GSH) (6), urate (5), etc. In addition, there are 3 main enzymes that carry sperm protection: catalase (CAT), superoxide dismutase (SOD), and glutathione peroxidase (GPx) (7). The metal ions copper and zinc are cofactors of SOD, iron is a co-factor of CAT, and selenium is a co-factor of GPx. Therefore, the availability of these metals in the organism plays a beneficial role, because they influence the activity of the corresponding enzymes. However, iron and copper could act as prooxidants, catalysing the formation of hydroxyl $(\mathrm{OH})$ radicals from superoxide anion radicals $\left(\mathrm{O}_{2}-\right)$ and hydrogen peroxide $\left(\mathrm{H}_{2} \mathrm{O}_{2}\right)(7,8)$. Hydroxyl radicals are highly reactive with a very short half-life and an extremely limited diffusion capacity. In practice, they attack and oxidize the first molecule they meet. Today it is assumed that $\mathrm{OH}$ have the most damaging effect on cell structures. The most vulnerable to oxidative attack are plasma membranes, due to the high percentage of polyunsaturated fatty acids in their composition. It has been suggested that the peroxidation of membrane lipids is largely responsible for sperm dysfunction (8).

This dual role of metal ions provokes our interest in investigating the concentration of copper, zinc, iron, and selenium in normal and pathological human semen and their impact on oxidative stress occurrence, evaluated by the levels of malondialdehyde (MDA) and GSH.

\section{MATERIALS AND METHODS}

\section{Chemicals}

All reagents and chemicals used for biochemical analyses [2-thiobarbituric acid (TBA), trichloroacetic acid (TCA), $\mathrm{HCl}$, reduced GSH and oxidized glutathione (GSSG), 5,5'-Dithiobis(2nitrobenzoic acid) (DTNB), GSH reductase] were obtained from Sigma-Aldrich Chemie GmbH (Darmstadt, Germany).

\section{Statement}

This research was performed in accordance with the Declaration of Helsinki for Human Researches [World Medical Association Declaration of Helsinki - Ethical principles for medical research involving human subjects. 59th WMA General Assembly, Seoul, Republic of Korea (2008)]. The approval of the Ethics Committee of the Research Board was obtained.

\section{Patients and semen collection}

Semen samples were obtained from men aged between 24 and 38 years attending a fertility clinic. Ejaculates were collected in sterile containers via masturbation after a sexual abstinence of 2 to 7 days. All participants were previously informed in detail about the objectives, tasks, and conduct of the survey. A written consent for participation in the research was taken from all patients.

\section{Semen parameter analysis}

After liquefaction of the semen samples for 30 minute at $37^{\circ} \mathrm{C}$ a standard analysis was performed within 1 hour according to World Health Organization guidelines (9) using a computer-assisted sperm class analyzer (Microptic SL, Barcelona, Spain). The software settings were those recommended by the manufacturer for the analysis of human sperm. Semen volume $(\mathrm{mL})$, count $\left(10^{\wedge} 6 / \mathrm{mL}\right)$, and motility $(\%)$ were included in spermiograms.

To determine the normal and respectively abnormal morphology (\%) of spermatozoa a SpermBlue staining method (Microptic SL, Barcelona, Spain) was applied. Ten $\mu \mathrm{L}$ of the respective ejaculates were pipetted onto slides. The smear was allowed to dry at room temperature. After that, the sample was placed in a fixing solution for 10 minute, and then stained in SpermBlue for 12-15 min. The morphology of at least 200 spermatozoa was analysed using the sperm class analyzer (Microptic SL, Barcelona, Spain) and a Leica DM 1000 microscope. Sperm morphology was assessed using 100x objective (immersion oil lens). Kruger's criteria, where morphology $<14 \%$ is considered abnormal, were used for evaluating spermatozoa morphology (10).

The samples were then categorized according to their seminal fluid analysis parameters into the following groups: normozoospermic, teratozoospermic (with normal morphology $<14 \%$ ), asthenoteratozoospermic (with motility $<40 \%$ and normal 
morphology $<14 \%$ ), and oligoteratozoospermic (with sperm concentration $<15$ million/mL and normal morphology $<14 \%$ ).

After semen analysis, the ejaculates were centrifuged at $3500 \mathrm{rpm}$ for 15 minute to separate the seminal plasma from spermatozoa. The obtained fractions were stored at $-80^{\circ} \mathrm{C}$ until biochemical and elemental analyses.

\section{Measurement of lipid peroxidation}

Lipid peroxidation was assessed by the quantity of the TBA reacting substances (TBARs), formed in the preparations, according to the method of Hunter et al. (11). To the semen samples $(0.5$ $\mathrm{mL}$ ) were added $0.3 \mathrm{~mL}$ of mixture, containing $2.8 \% \mathrm{TCA}+5 \mathrm{M}$ $\mathrm{HCl}+2 \%$ TBA in $50 \mathrm{mM} \mathrm{NaOH}(2: 1: 2 \mathrm{v} / \mathrm{v})$, which were heated at $100{ }^{\circ} \mathrm{C}$ for 15 minutes for color development. The samples were cooled and centrifuged at $1000 \mathrm{x}$ g for 10 minutes. Then the resulting supernatant was separated, and the absorbance was read at $532 \mathrm{~nm}$ and $600 \mathrm{~nm}$ against appropriate blanks. The 600 $\mathrm{nm}$ absorbance was subtracted from that of $532 \mathrm{~nm}$, because the former was considered to be a non-specific baseline. Every assay was conducted in triplicate using a Jenway 6305 Single Beam UV/Visible spectrophotometer (Bibby Scientific Ltd., Stone, Staffordshire, UK). The values were expressed in nmols MDA/ $\mathrm{mL}$, using a molar extinction coefficient of $1.56 \times 10^{\wedge} 5 \mathrm{M}^{-1} \mathrm{~cm}^{-1}$.

\section{Determination of total glutathione level}

The total GSH (tGSH) was measured spectrophotometrically by the method of Akerboom and Sies (12) using DTNB. This colorimetric assay is based on the reaction between GSH and DTNB where TNB (5-thio-2-nitrobenzoic acid) is formed. TNB exhibits maximum absorbance at $412 \mathrm{~nm}$. The intensity of the absorbance is proportional to the GSH level in the sample. A standard curve, prepared with known amounts of GSH, was used for determining the sample's GSH concentrations. Every assay was conducted in triplicate using a Jenway 6305 Single Beam UV/Visible spectrophotometer (Bibby Scientific Ltd., Stone, Staffordshire, UK). The values were expressed in $\mu \mathrm{mol} / \mathrm{L}$.

\section{Determination of $\mathrm{Fe}, \mathrm{Cu}, \mathrm{Zn}$, and Se levels}

The iron, copper, zinc, and selenium concentrations in sperm were estimated by atomic absorption spectrophotometry, using a Perkin Elmer Zeeman 5100PC (Perkin-Elmer GmbH, Rodgau, Germany) provided with an HGA 600 graphite furnace programmer. Seminal samples were diluted 1:50 with deionized water to determine the zinc concentration and were directly measured by flame atomic spectroscopy, whereas a graphite furnace atomic absorption spectroscopy technique was used to assess copper, iron, and selenium after 1:10 dilution with deionized water. For quality control, standard materials (Merck, Darmstadt, Germany) were used as references.

\section{Statistical analysis}

Values were presented as mean \pm standard deviation. For establishing the differences between groups an analysis of variance (one-way ANOVA) was performed, and for conducting pairways comparisons the post hoc test (Tukey test) was applied. At values of $p<0.05$, the differences were considered to be significant. For data analysis the Windows computing program Statistical Package for the Social Sciences "SPSS 16" (SPSS Inc., Chicago, IL, USA) was used.

\section{RESULTS}

The average values of the sperm specimen parameters from the four investigated groups are given in Table 1. A significant decrease in the semen count, motility, and percentage of normal morphology was observed in the three abnormal groups when compared to normozoospermics; there were no significant differences in seminal volume.

Significantly elevated levels of MDA were detected in the abnormal groups, as in the teratozoospermic group the level was increased threefold and in the asthenoteratozoospermic group fourfold in comparison to the normozoospermic group. The detected level of tGSH was almost twice lower in the asthenoteratozoospermic than in the normozoospermic group (Table 2). There were no significant differences between the tGSH level of the teratozoospermic and oligoteratozoospermic group.

The concentrations of the seminal trace elements are presented in Table 3. The concentration of $\mathrm{Zn}$ and Se were significantly lower in the three abnormal groups than in the control group. In

TABLE 1. Patients' age and sperm parameters in the groups

\begin{tabular}{|c|c|c|c|c|}
\hline & $\begin{array}{l}\text { Normo-zoospermia } \\
\qquad(\mathrm{n}=6)\end{array}$ & $\begin{array}{l}\text { Terato-zoospermia } \\
\qquad(\mathrm{n}=6)\end{array}$ & $\begin{array}{l}\text { Asthenoterato-zoospermia } \\
\qquad(\mathrm{n}=6)\end{array}$ & $\begin{array}{l}\text { Oligoterato-zoospermia } \\
\qquad(\mathrm{n}=6)\end{array}$ \\
\hline Age (years) & $36.00 \pm 4.55$ & $30.50 \pm 4.60$ & $35.50 \pm 0.35$ & $37.02 \pm 3.35$ \\
\hline Seminal fluid volume (mL) & $2.53 \pm 0.93$ & $2.90 \pm 0.07$ & $3.03 \pm 0.69$ & $3.29 \pm 0.56$ \\
\hline Sperm count (million/mL) & $101.12 \pm 18.9$ & $61.98 \pm 16.38^{*}$ & $25.66 \pm 3.09 *$ & $8.57 \pm 0.43^{*}$ \\
\hline Sperm motility (\%) & $72.89 \pm 6.79$ & $59.56 \pm 4.98 *$ & $37.85 \pm 3.01 *$ & $50.47 \pm 5.49 *$ \\
\hline Sperm morphology/pathological forms (\%) & $39.29 \pm 2.25$ & $98.01 \pm 4.71 *$ & $90.75 \pm 5.94 *$ & $93.79 \pm 3.15^{*}$ \\
\hline
\end{tabular}


TABLE 2. Concentration of malondialdehyde and total glutathione in seminal plasma in the groups

\begin{tabular}{|c|c|c|c|c|}
\hline & $\begin{array}{l}\text { Normo-zoospermia } \\
\qquad(\mathrm{n}=6)\end{array}$ & $\begin{array}{l}\text { Terato-zoospermia } \\
\qquad(\mathrm{n}=6)\end{array}$ & $\begin{array}{l}\text { Asthenoterato-zoospermia } \\
\qquad(n=6)\end{array}$ & $\begin{array}{l}\text { Oligoterato-zoospermia } \\
\qquad(n=6)\end{array}$ \\
\hline MDA nmol/mL & $0.92 \pm 0.06$ & $2.57 \pm 0.20 *$ & $3.30 \pm 0.35^{*}$ & $1.26 \pm 0.14^{*}$ \\
\hline $\mathrm{tGSH} \mu \mathrm{mol} / \mathrm{L}$ & $78.13 \pm 7.00$ & $63.50 \pm 3.18^{*}$ & $36.10 \pm 7.14^{*}$ & $56.00 \pm 8.23 *$ \\
\hline
\end{tabular}

TABLE 3. Concentration of iron, copper, zinc, and selenium in seminal plasma in the groups

\begin{tabular}{|c|c|c|c|c|}
\hline & $\begin{array}{l}\text { Normo-zoospermia } \\
\qquad(\mathrm{n}=6)\end{array}$ & $\begin{array}{l}\text { Terato-zoospermia } \\
\qquad(\mathrm{n}=6)\end{array}$ & $\begin{array}{l}\text { Asthenoterato-zoospermia } \\
\qquad(\mathrm{n}=6)\end{array}$ & $\begin{array}{l}\text { Oligoterato-zoospermia } \\
\qquad(\mathrm{n}=6)\end{array}$ \\
\hline Iron $\mathrm{mg} / \mathrm{L}$ & $2.70 \pm 0.19$ & $3.05 \pm 0.24$ & $4.25 \pm 0.53 *$ & $2.90 \pm 0.30$ \\
\hline Copper mg/L & $136.33 \pm 10.71$ & $126.00 \pm 11.31$ & $144.00 \pm 2.83$ & $120.20 \pm 15.32$ \\
\hline Zinc $\mathrm{mg} / \mathrm{L}$ & $150.67 \pm 4.75$ & $127.00 \pm 12.37 *$ & $127.50 \pm 5.30 *$ & $120.40 \pm 13.98^{*}$ \\
\hline Selenium $\mu \mathrm{g} / \mathrm{L}$ & $72.00 \pm 3.86$ & $61.98 \pm 6.38 *$ & $60.00 \pm 3.54 *$ & $59.00 \pm 5.40 *$ \\
\hline
\end{tabular}

Values are expressed as mean \pm standard deviation; ${ }^{*} \mathrm{p}<0.05$ compared to normozoospermic group

regard to Fe concentrations, they were higher in the abnormal groups, but a significant difference was registered only between the asthenoteratozoospermic group and normozoospermic group $(4.25 \pm 0.53$ vs $2.70 \pm 0.19 \mathrm{mg} / \mathrm{L})$. We did not find significant differences in the level of $\mathrm{Cu}$ between the tested groups.

From the studied trace elements, only Fe demonstrated a significant positive correlation with the MDA level $(\mathrm{r}=0.666$, $\mathrm{p}=0.018)$ and a negative significant correlation with the $\mathrm{tGSH}$ level $(\mathrm{r}=-0.689, \mathrm{p}=0.013)$. The seminal tGSH level correlated positively with sperm motility $(\mathrm{r}=0.589, \mathrm{p}=0.044)$.

\section{DISCUSSION}

Trace elements play an essential role in all aspects of human physiology, including reproduction. Metal ions, among their many other functions, play essential roles in about one-third of enzymes (13). In terms of the pro-/antioxidant balance in the organism, the metal ions copper, zinc, iron, and selenium are very important, acting as co-factors of the antioxidant enzymes $\mathrm{Cu}, \mathrm{Zn}-\mathrm{SOD}, \mathrm{CAT}$, and GPx, respectively. Thus their availability in the organism should play a beneficial role, because their presence determines the respective antioxidant enzyme activity. However, iron and copper could act as prooxidants, inducing oxidative damage of the cell compounds. On the one hand, iron is a co-factor of catalase, the enzyme that catalyses the decomposition of hydrogen peroxide. Many authors have recognized $\mathrm{H}_{2} \mathrm{O}_{2}$ as the most toxic oxidizing agent for human spermatozoa (3). Therefore the decomposition of $\mathrm{H}_{2} \mathrm{O}_{2}$ is essential for preventing its toxic effect. Khosrowbeygi et al. (14) found a significantly lower catalase activity in patients with asthenozoospermia, asthenoteratozoospermia, and oligoasthenoteratozoospermia than in normozoospermic males. They concluded that the decreased seminal plasma catalase activity could be associated with poor sperm quality. On the other hand, it is well known that transition metal ions such as iron and copper can generate the highly reactive hydroxyl radicals in the presence of $\mathrm{H}_{2} \mathrm{O}_{2}$ and $\mathrm{O}_{2}$ - (Haber-Weiss reaction) or $\mathrm{H}_{2} \mathrm{O}_{2}$ (Fenton reaction) (7). A high concentration of iron has been found in whole semen - about $500 \mu \mathrm{g} \%$ - and the concentration in seminal plasma was reported as $315 \mu \mathrm{g} \%$ (between 265 and $365 \mu \mathrm{g} \%$ ) (15), both obtained from normal fertile men. In comparison, the total iron in blood plasma is about $100 \mu \mathrm{g} \%$ (16). Our findings correspond to the above-mentioned concentrations with a mean value of $270 \mu \mathrm{g} \%(2.7 \mathrm{mg} / \mathrm{L})$ iron in the seminal plasma. We found an increase of iron levels in the asthenoteratozoospermic versus the normozoospermic group, but there was no significant correlation between iron concentration and either sperm motility, morphology, or concentration. Skandhan et al. (15) reported cases with a much higher iron concentration, accompanied by motility of less than $10 \%$. Huang et al. (17) also detected higher levels of iron in asthenospermic men than in normal controls, which were accompanied by higher levels of MDA. The authors observed a negative correlation between the MDA concentration and sperm motility in the abnormal groups $(\mathrm{r}=-0.28, \mathrm{p}<0.05)$, but with no association among trace elements and MDA in seminal plasma. The incubation of human sperm with $\mathrm{Fe}^{2+}$ led to a significant reduction of the sperm motility at $5 \mathrm{ppm}$ and an increase of MDA levels (18). These results suggest that increased lipid peroxidation in response to elevated $\mathrm{Fe}^{2+}$ levels may inhibit sperm motility. Our findings showed a significant positive correlation between the iron concentration in the samples and the lipid peroxidation, as well as a negative correlation between iron concentration and the tGSH level. The high MDA level and low tGSH level could be one of the causes of decreased motility and increased percentage of abnormal spermatozoa. MDA is an end product of lipid peroxidation and serves as an important tool in the 
measurement of lipid peroxidation. It is well known that spermatozoa are more susceptible to lipid peroxidation than other cells. There are two reasons for this: 1) during the final stages of spermatogenesis they discard most of their cytoplasm, along with cytoplasmic defence enzymes like CAT and SOD, and 2) their plasma membrane is rich in polyunsaturated fatty acids. There is a lot of evidence that MDA concentration is negatively associated with sperm motility, concentration, and normal morphology, and exhibits an inverse relationship with sperm-oocyte fusion (19). GSH is considered to be a major antioxidant within the cells. It is a co-factor required for the activity of GPx, which directly binds to ROS via its free sulphydryl groups. The presence of GSH in the extracellular space has a protective effect on the sperm plasma membrane, since it can detoxify cytotoxic aldehydes generated during lipid peroxidation (20). Significantly higher GSH levels have been detected in normozoospermic men than in groups with sperm defects and a significant relationship between seminal GSH and sperm motility (20) has been suggested - observations similar to our results. In turn, GPx is a selenoprotein and in fact the importance of Se in mammals is due to its role as a prosthetic group in the catalytic sites of the enzyme. Although we did not find any correlations between Se content, sperm parameters, and oxidative status, many authors have demonstrated a negative effect of low levels of seminal Se on the number and motility of spermatozoa (20). It has been shown that both Se (21) and Zn (22) play an essential role in testicular growth processes, testosterone secretion, and spermatogenesis. $\mathrm{Zn}$ concentration is high in seminal plasma and exceeds the blood mean values 30 times (23). Significant differences in seminal $\mathrm{Zn}$ concentrations have been detected between fertile and infertile men. This metal is a co-factor of $\mathrm{Cu}, \mathrm{Zn}-\mathrm{SOD}$ and it was supposed that the reduction in $\mathrm{Zn}$ concentration disrupted antioxidant defence, making the spermatozoa more susceptible to oxidative damage of membrane lipids, proteins, and DNA (24). Therefore the positive relationship between increased seminal $\mathrm{Zn}$ content and sperm production, motility, and morphology is reasonable. In fact, in the molecule of $\mathrm{Cu}$, $\mathrm{Zn}-\mathrm{SOD}, \mathrm{Zn}^{2+}$ has a structural, stabilizing role, while in the catalytic activity $\mathrm{Cu}^{2+}$ is directly involved. SOD is one of the most important antioxidative defence enzymes. It catalyses the conversion of superoxide radicals to $\mathrm{H}_{2} \mathrm{O}_{2}$, which in turn could be degraded by GPx or catalase to the harmless oxygen molecule and water. This mechanism prevents the lipid peroxidation of the plasma membrane. If it is impossible to eliminate $\mathrm{H}_{2} \mathrm{O}_{2}$ from the cell, it can interact with a trace metal ion and generate the highly reactive $\mathrm{OH}$ radicals. Therefore the action of SOD should be conjugated with that of catalase or GPx to prevent the $\mathrm{H}_{2} \mathrm{O}_{2}$ action (25).
In regard to copper concentration, a number of animal and human studies found an inverse association between high $\mathrm{Cu}$ levels and semen quality $(17,26)$. On the other hand, the concentration of $\mathrm{Cu}$ could influence the activity of coppercontaining enzymes such as SOD. Abdul Rasheed (27) suggested a direct relationship between the level of copper in seminal plasma and the activity of SOD. In azoospermic patients he observed a significant decrease in the levels of seminal plasma copper that may lead to a concomitant decrease in SOD activity. Thus the decreased copper content may be considered an important mechanism of increased oxidative stress in azoospermic semen. Therefore maintenance of the microelements balance is crucial because both deficiency and excess may have negative consequences.

The studied elements ( $\mathrm{Fe}, \mathrm{Cu}, \mathrm{Zn}$, and $\mathrm{Se}$ ) belong to the group of microminerals. Along with vitamins, they are regarded as the main class of micronutrients in the diet. Several studies have reported an improvement of sperm quality and increase of pregnancy rates when men changed the diet or took certain vitamins and micronutrients (28). Therefore a better comprehension of the trace elements' mechanism of action and their effects on male fertility would contribute to the medication of male infertility. The levels of trace elements in the normozoospermic group as well as in various categories of abnormal sperm should be well identified, because both a deficiency and excess of these elements can disrupt the biological equilibrium and impact on fertility. Respectively an imbalance on one side or another could be restored with appropriate treatment. We detected a nearly significant correlation between age and $\mathrm{Se}(\mathrm{r}=-0.545, \mathrm{p}=0.067)$, as well as between $\mathrm{Zn}$ and morphology $(\mathrm{r}=-0.513, \mathrm{p}=0.088)$, that required a further study with a larger number of people.

In conclusion, our results established alterations in the concentration of iron, zinc, and selenium in abnormal groups compared to normozoospermic group. These trace elements act as co-factors of the antioxidant enzymes CAT, SOD, and GPx, respectively, and their availability could affect the oxidative status of sperm. In addition, iron could act as a prooxidant via Fenton reaction. The impaired oxidative balance of spermatozoa could contribute to poor sperm motility and morphology, since in all abnormal groups an increased lipid peroxidation and decreased tGSH levels were detected. We found a significant correlation between the Fe levels and the tested markers of oxidative stress. Further research on the relationship between antioxidant enzyme activities and the level of their trace elemental co-factors in semen as well as their impact on the sperm parameters is required. Improved knowledge about the role of trace metals in male infertility could be useful in overcoming this serious problem in our contemporary life. 
Financial Disclosure: No financial disclosure was declared by the authors.

Conflict of Interest: No conflict of interest was declared by the authors.

\section{REFERENCES}

1. Boivin J, Bunting L, Collins JA, Nygren KG. International estimates of infertility prevalence and treatment-seeking: potential need and demand for infertility medical care. Human Reprod 2007;22:1506-12.

2. Agarwal A, Prabakaran SA. Mechanism, measurement, and prevention of oxidative stress in male reproductive physiology. Indian J Exp Biol 2005;43:963-74.

3. Aitken RJ, Buckingham D, Harkiss D. Use of a xanthine oxidase free radical generating system to investigate the cytotoxic effects of reactive oxygen species on human spermatozoa. J Reprod Fertil 1993;97:441-50.

4. Gallardo JM. Evaluation of antioxidant system in normal semen. Rev Invest Clin 2007;59:42-7.

5. Gavella M, Lipovac V, Vučić M, Ročić B. Evaluation of ascorbate and urate antioxidant capacity in human semen. Andrologia 1997;29:29-35.

6. Bhardwaj A, Verma A, Majumdar S, Khanduja KL. Status of vitamin E and reduced glutathione in semen of oligozoospermic and azoospermic patients. Asian J Androl 2000;2:225-8.

7. Agarwal A, Saleh RA. Role of oxidants in male infertility: rationale, significance, and treatment. Urol Clin North Am 2002;29:817-27.

8. Griveau, JF, Dumont E, Renard B, Callegari JP, Lannou D. Reactive oxygen species, lipid peroxidation and enzymatic defense systems in human spermatozoa. J Reprod Fertil 1995;103:17-26.

9. World Health Organization. WHO laboratory manual for the examination and processing of human semen - 5th ed. Geneva, $\mathrm{CH}$ : World Health Organization; 2010.

10. Kruger TF, Acosta AA, Simmons KF, Swanson RJ, Matta JF, Oehringer S. Predictive value of abnormal sperm morphology in in vitro fertilization. Fertil Steril 1988;49:112-7.

11. Hunter F Jr, Gebinski JM, Hoffstein PE, Weinstein J, Scott A. Swelling and lysis of rat liver mitochondria by ferrous ions. J Biol Chem 1963;238:82835 .

12. Akerboom TP, Sies H. Assay of glutathione, glutathione disulfide, and glutathione mixed disulfides in biological samples. Methods Enzymol 1981;77:373-82.
13. Silva J. J. R. F. D., Williams R. J. P. The Biological Chemistry of the Elements, Clarendon Press: Oxford; 1991.

14. Khosrowbeygi A, Zarghami N, Deldar Y. Correlation between sperm quality parameters and seminal plasma antioxidants status. Iranian Journal of Reproductive Medicine 2004;2:58-64.

15. Skandhan KP, Mazumdar BN, Sumangala B. Study into the iron content of seminal plasma in normal and infertile subjects. Urologia 2012;79:54-7.

16. Charlton RW, Bothwell TH. In: Thompson RGS Woodton IPP, Eds. Idiopathic haemochromatosis and related iron storage disorders. Biochemical disorders in human disease, 3-rd edition J \& A Churchill, London; 1970:201-14.

17. Huang YL, Tseng WS, Cheng SY, Lin TH. Trace elements and lipid peroxidation in human seminal plasma. Biol Trace Elem Res 2000;76:20715.

18. Huang YL, Tseng WC, Lin TH. In vitro effects of metal ions (Fe2+, Mn2+, $\mathrm{Pb} 2+)$ on sperm motility and lipid peroxidation in human semen. J Toxicol Environ Health A 2001;62:259-67.

19. Aitken RJ, Clarkson JS, Fishel S. Generation of reactive oxygen species, lipid peroxidation, and oxygen species, lipid peroxidation, and human sperm function. Biol Reprod 1989;41:183-97.

20. Atig F, Raffa M, Habib BA, Kerkeni A, Saad A, Ajina M. Impact of seminal trace element and glutathione levels on semen quality of Tunisian infertile men. BMC Urol 2012;12:6.

21. Akinloye O, Arowojolu AO, Shittu OB, Adejuwon CA, Osotimehin B. Selenium status of idiopathic infertile Nigerian males. Biol Trace Elem Res 2005;104:9-18.

22. Colagar AH, Marzony ET, Chaichi MJ. Zinc levels in seminal plasma are associated with sperm quality in fertile and infertile men. Nutr Res 2009;29:82-8

23. Xu B, Chia SE, Tsakok M, Ong CN. Trace elements in blood and seminal plasma and their relationship to sperm quality. Reprod Toxicol 1993;7:613708

24. Powell SR. Antioxidant properties of zinc. J Nutr 2000;130:1447-54

25. Shiva M, Gautam AK, Verma Y, Shivgotra V, Doshi H, Kumar S. Association between sperm quality, oxidative stress, and seminal antioxidant activity. Clin Biochem 2011;44:319-24.

26. Skandhan KP. Review on copper in male reproduction and contraception Rev Fr Gynecol Obstet 1992;87:594-8.

27. Abdul Rasheed OF. Association between seminal plasma copper and magnesium levels with oxidative stress in Iraqi infertile men. Oman Med J 2010;25:168-72

28. Buhling KJ, Laakmann E. The effect of micronutrient supplements on male fertility. Curr Opin Obstet Gynecol 2014;26:199-209. 